FORMATION Formation emploi

Revue française de sciences sociales

93 | janvier-mars 2006

Au-delà du genre, l'insertion

\title{
Les salaires des hommes et des femmes en début de vie active : des sources de disparité variables selon les professions
}

Men's and women's wages at the start of their careers: the reasons for the disparities depend on the gender segregation inherent to their occupations Einkommen von Frauen und Männern beim Eintritt ins Berufsleben:

Lohnungleichheiten innerhalb der Berufsgruppen

Thomas Couppié, Arnaud dupray et Stéphanie Moullet

\section{OpenEdition}

Journals

Édition électronique

URL : http://journals.openedition.org/formationemploi/2320

DOI : $10.4000 /$ formationemploi.2320

ISSN : 2107-0946

Éditeur

La Documentation française

Édition imprimée

Date de publication : 1 mars 2006

Pagination : 29-47

ISSN : 0759-6340

Référence électronique

Thomas Couppié, Arnaud dupray et Stéphanie Moullet, «Les salaires des hommes et des femmes en début de vie active : des sources de disparité variables selon les professions », Formation emploi [En ligne], 93 | janvier-mars 2006, mis en ligne le 03 décembre 2009, consulté le 30 octobre 2020. URL http://journals.openedition.org/formationemploi/2320 ; DOI : https://doi.org/10.4000/

formationemploi.2320 


\section{Les salaires des hommes et des femmes en début de vie active : des sources de disparité variables selon les professions}

Par Thomas Couppié, Arnaud Dupray et Stéphanie Moullet*

Les jeunes femmes sont plus diplômées; pourtant, elles sont aussi moins rémunérées. Dans quelle mesure sont-elles l'objet d'une discrimination salariale? Le constat peut différer selon que la profession est plutôt à dominante masculine, féminine ou mixte et selon que cette dominante a résulté d'une ségrégation scolaire ou s'est plutôt construite sur le marché du travail.

Les disparités de salaire et de carrière entre hommes et femmes sont l'objet, depuis deux décennies, de nombreuses études tant en économie qu'en sociologie. Ces travaux ont largement mis en évidence le handicap salarial subi par les femmes sur le marché du travail. Dans la voie initiée par Blinder (1973) et Oaxaca (1973), des études récentes ont pointé que celui-ci prend effet très tôt, dès les premières années de vie active (Havet et Lacroix, 2002; Dupray et Moullet, 2004). Ainsi, la valorisation monétaire des caractéristiques individuelles et d'emploi se révèle inférieure pour les femmes sur le marché du travail, et vient renforcer ${ }^{1}$ les effets salariaux provoqués par des différences de situation.
* Thomas Couppié est démographe, chargé d'études au Département des entrées dans la vie active du Centre d'études et de recherches sur les qualifications (Céreq). II travaille sur les disparités d'insertion entre hommes et femmes et sur les questions de ségrégation professionnelle, sur la correspondance emploi-formation dans le tertiaire et l'insertion comparée des jeunes en Europe. II a notamment publié avec Michèle Mansuy: "L'insertion professionnelle des débutants en Europe : des situations contrastées » Économie \& Statistique n 378-379, 2004 et avec Alberto Lopez et Jean-François Giret: " Des formations initiales aux premiers emplois : une correspondance plutôt mal assurée », in Des formations pour quels 
emplois ?, Giret J.-F., Lopez A., Rose J. (dir.), Éditions La Découverte, 2005.

Arnaud Dupray est économiste, chargé d'études au Département professions et marché du travail du Céreq. Ses thématiques de recherche portent sur la mobilité professionnelle et les conditions d'évolution de carrière, notamment selon le genre. Parmi ses dernières publications, "Les mobilités en début de vie professionnelle ", Bref-Céreq, $n^{\circ} 216,2005$; en collaboration avec S. Moullet, "Les salaires des hommes et des femmes", Bref- Céreq, $n^{\circ} 219,2005$; en collaboration avec $\mathrm{L}$. Diederichs-Diop, "Trajectoire initiale et devenir professionnel en seconde partie de carrière » dans M-E Joël J. Wittwer (coord.) Économie du vieillissement, âge et emploi, L'Harmattan, 2005, pp. 251-271.

Stéphanie Moullet est économiste, chargée d'études au Département des entrées dans la vie active du Céreq. Elle est l'auteur d'une thèse sur les rendements des investissements en éducation. Elle conduit aussi des travaux sur les disparités de carrières des hommes et des femmes. Elle a notamment publié : "Externalités de l'éducation et mobilité intergénérationnelle : application au cas français " (avec A. Fabre), Économie et Prévision, 5/166, 2004. "Ségrégation professionnelle et discrimination salariale entre hommes et femmes en début de vie active » (avec Couppié T. et Dupray A.) in L'insertion professionnelle des femmes - Entre contraintes et stratégies d'adaptation, Presses universitaires de Rennes, 2006. "Femmes à l'entrée du marché du travail: un retard salarial en partie inexpliqué », (avec. Dupray A.), Céreq, Note Emploi Formation, n 12, 2004.

Cependant, les travaux basés sur ces modèles n'ont que partiellement intégré l'hétérogénéité des différents segments du marché du travail. En particulier, les relations entre, d'une part, la nature des écarts de salaires entre hommes et femmes et, d'autre part, les caractéristiques des différentes professions ne sont que depuis peu abordées dans la littérature (Meng et Meurs, 2001 pour la France ; Baker et Fortin, 2001 ; Bayard et al., sous presse). Or, pour certaines analyses récentes, les inégalités d'insertion entre hommes et femmes varient non seulement selon « le genre » de la profession (et donc le degré de ségrégation par sexe qui la caractérise), mais aussi selon l'origine de cette ségrégation
(Couppié et Epiphane, 2004). On entend par origine de la ségrégation le fait que celle-ci peut soit provenir de la ségrégation éducative (la profession recrutant dans des formations sexuellement clivées à l'exemple des infirmières) soit résulter des mécanismes d'appariement s'opérant sur le marché du travail entre individus et emplois sexuellement différenciés, indépendamment des qualifications scolaires acquises (une profession accordant, à formation donnée, la préférence aux candidats d'un seul sexe, à l'exemple des secrétaires). Cette approche suggère que pour certaines professions dont la ségrégation est principalement d'origine éducative, les diplômes joueraient un rôle plus important dans les recrutements et les déroulements de carrière, laissant dès lors moins de place à la prise en compte d'autres critères, dont le sexe.

De manière originale, nous utilisons une répartition des professions selon leur ségrégation pour tester un certain nombre d'hypothèses portant non seulement sur l'intensité des écarts de rémunération entre hommes et femmes, mais également sur l'importance de la composante non expliquée de ces écarts, que l'on assimile usuellement à la discrimination. Les hypothèses formulées seront examinées sur la base de dix groupes professionnels définis selon leur degré de ségrégation, selon l'origine - éducative ou liée au marché du travail - de celle-ci et selon qu'il s'agit d'emplois du sommet de la hiérarchie sociale (niveaux cadre et profession intermédiaire) ou de la base de cette hiérarchie (niveaux employé et ouvrier).

L'enjeu de l'analyse est de repérer les groupes professionnels au sein desquels les femmes seraient plus particulièrement discriminées du point de vue salarial; ce repérage pouvant orienter les politiques publiques dans le domaine du droit des femmes. En effet, si le groupe professionnel qui se distingue par l'importance de la discrimination se caractérise par une ségrégation d'origine éducative - et sous l'hypothèse que ces ségrégations, scolaire puis professionnelle, ne constituent pas un motif d'orientationalors une politique publique agissant sur la diversification des orientations scolaires des filles et des garçons serait efficace. S'il s'avère que la mixité des emplois est gage d'une moindre discrimination salariale à l'encontre des femmes, l'action publique peut alors passer non seulement par des incitations à la diversification des choix de filières pour les filles comme pour les garçons, mais aussi par des incitations auprès des employeurs en faveur de cette mixité. 
À partir d'une enquête d'insertion professionnelle (Génération 98 du Céreq), nous comparons entre chaque groupe professionnel, les décompositions des écarts de salaire entre hommes et femmes, en distinguant la composante liée aux différences de caractéristiques individuelles et d'emplois de ce qui relève de différences de valorisation monétaire de caractéristiques identiques.

\section{UNE RÉPARTITION DES PROFESSIONS SELON L'ORIGINE DE LA SEGRÉGATION PROFESSIONNELLE}

Nous faisons l'hypothèse ici que la discrimination salariale (voir encadré 1) n'est pas un phénomène homogène entre les différents segments professionnels du marché du travail. L'intensité et la source de la ségrégation professionnelle - d'origine éducative ou liée au marché du travail - observées entre hommes et femmes sont la clé d'entrée de notre segmentation des professions.

La distinction entre ségrégations d'origine éducative et liée au marché du travail découle de l'idée que l'inégale répartition des hommes et des femmes dans les professions trouve à la fois sa source dans le capital scolaire différencié des unes et des autres et dans des mécanismes ségrégatifs d'allocation sur le marché du travail, à capital scolaire donné. On peut traduire algébriquement cette idée (Couppié et Epiphane, 2004, 2006) en décomposant en deux termes l'indice de ségrégation professionnelle proposé par Karmel et Maclachlan (1988) ; cet indice récapitule la proportion d'individus qu'il faudrait déplacer afin d'égaliser les distributions des hommes et des femmes dans les professions. On arrive ainsi à trois indices de ségrégation qu'on peut décliner pour chacun des groupes professionnels : un indice général décomposé en deux sous-indices additifs représentant respectivement la ségrégation d'origine éducative et celle prenant corps sur le marché du travail. En fonction des valeurs prises par ces trois indices, Couppié et Epiphane $(2004,2006)$ proposent une typologie des professions. D'une part, ils isolent les professions les plus faiblement ségréguées (et donc « mixtes ») des autres. D'autre part, pour les professions plus fortement ségréguées (donc "féminines》 ou «masculines »), ils opposent celles dont la ségrégation est principalement d'origine éducative aux autres, c'est-à-dire à celles dont la ségrégation est aussi ou principalement liée au marché du travail. On arrive alors à une typologie distinguant cinq groupes de profession ${ }^{2}$.

En outre, il semble opportun de différencier les professions selon leur niveau de qualification. On sait en effet que les cadres sont susceptibles d'être davantage touchés que les employés ou ouvriers par des mécanismes d'individualisation des rémunérations. Ces mécanismes tendent à ouvrir l'éventail des critères à la source des variations de rémunération entre hommes et femmes, de manière distincte de ce qu'on peut observer dans les autres catégories socioprofessionnelles. Chez les ouvriers et employés, certains écarts de situation plus flagrants comme les proportions d'hommes et de femmes travaillant à temps partiel, peuvent contribuer plus fortement aux écarts de rémunération que dans les qualifications de niveau supérieur. À ces raisons d'ordre économique s'ajoute un motif technique lié au risque d'effets de composition si, par exemple, dans certains types de professions masculines, les femmes sont concentrées dans les niveaux intermédiaires et supérieurs de la hiérarchie des qualifications. L'écart constaté dans celles-ci ne proviendrait alors pas tant de la ségrégation attachée à l'univers professionnel considéré que du fait qu'hommes et femmes n'y occupent pas les mêmes niveaux de qualification.

De manière pratique, ne pouvant multiplier à loisir les catégories compte tenu de la taille des échantillons, nous traitons la typologie en cinq groupes pour les cadres et professions intermédiaires, d'un côté, et pour les employés et ouvriers, de l'autre.

En résumé, les cadres et professions intermédiaires son rassemblés dans les catégories 1 à 5 , les ouvriers et employés dans les catégories 6 à 10. La nature ségréguée des professions se déclinant dans les deux cas de la même façon, les groupes professionnels obtenus sont présentés selon leur composition sexuée et l'origine de la ségrégation (voir tableau 1). Le tableau ne prétend pas à l'exhaustivité, les professions les plus emblématiques dans chaque catégorie sont données à titre d'illustration.

\footnotetext{
${ }^{2}$ Nous avons réduit le nombre de types à cinq (contre huit dans la typologie d'origine (Couppié et Epiphane, 2006)) en privilégiant l'opposition entre ségrégation principalement d'origine éducative et ségrégation aussi ou principalement liée au marché du travail.
} 


\section{Encadré 1}

\section{La discrimination vue par les économistes : théories et méthode}

La discrimination sexuelle sur le marché du travail a fait l'objet d'une vaste littérature économique, notamment anglo-saxonne (Altonji et Blank, 1999). Si l'on considère les caractéristiques relatives à l'éducation des individus à l'entrée du marché du travail comme exogènes à la discrimination économique, alors les mécanismes de discrimination empruntent essentiellement à trois phénomènes. Le premier a trait à la ségrégation professionnelle selon laquelle la population discriminée va se trouver concentrée dans des emplois dont les conditions de travail et de salaire sont plutôt défavorables. La discrimination est alors liée à l'affectation d'emploi, et l'écart de situation professionnelle explique alors la discrimination en termes de salaire. Le deuxième phénomène renvoie à une discrimination purement salariale qui passe, à emploi donné, par une valorisation monétaire différenciée des caractéristiques productives des individus. Enfin, une dernière forme de discrimination relèverait essentiellement des différences d'opportunité de mobilité ascendante et de formation continue proposées aux individus selon leur origine ou leur sexe et ce, à caractéristiques de leur emploi égales. Ces trois phénomènes sont susceptibles d'intervenir de manière autonome ou de se combiner. Une fois dans l'emploi, la discrimination s'exerce au travers de la valorisation monétaire différenciée des caractéristiques individuelles et d'emploi identiques. II s'agit d'une discrimination salariale si les écarts de valorisation monétaire sont systématiquement corrélés avec certaines caractéristiques non économiques des individus (Stiglitz, 1973), comme ici le sexe.

La mesure de la discrimination salariale, trois ans après la sortie de formation initiale, selon la nature des groupes professionnels en matière de ségrégation, permettra d'éclairer l'importance des deux premiers types de phénomènes.

L'analyse économétrique de la discrimination salariale repose sur une méthode de décomposition introduite par Blinder (1973) et Oaxaca (1973). Elle permet de départager ce qui, dans l'écart de salaire estimé aux caractéristiques moyennes des deux groupes d'individus, (i) est relatif à une différence dans les caractéristiques possédées par les individus selon leur sexe, (ii) se rapporte au traitement salarial différencié (selon le sexe) des mêmes caractéristiques. Cette seconde composante, non expliquée par les différences de caractéristiques observées des individus, est classiquement conçue comme la manifestation d'un phénomène de discrimination. Notons que l'on a retenu une structure de paiement non discriminante unique où l'on contrôle l'affectation dans chacun des groupes professionnels. Cette méthode permet en effet de comparer entre eux les différents groupes, c'est-à-dire les composantes liées aux différences de caractéristiques et aux différences de valorisation monétaire de ces caractéristiques, à la source des écarts de salaires.

L'analyse conduite ici laisse hors du modèle - et donc ne traite pas - les conditions d'affectation et de choix des individus dans l'ensemble des groupes professionnels, de même que les conditions d'orientation dans les études. Si certaines caractéristiques individuelles prises en compte dans la détermination des salaires contribuent également à orienter les individus dans l'éventail des professions, et notamment si certaines jouent différemment chez les hommes et les femmes, cette simplification peut conduire à ce que certains écarts de salaire établis entre hommes et femmes soient liés pour une part à une étape préalable du processus d'allocation des individus sur le marché du travail. Comme un tel approfondissement déborde du cadre imparti à cet article, cette simplification invite à interpréter avec prudence les résultats dégagés.

Les caractéristiques observées renvoient aussi bien aux caractéristiques individuelles acquises avant l'entrée sur le marché du travail qu'à celles relatives à l'emploi occupé (niveau de qualification, statut du contrat de travail...). 
Suite Encadré 1

Sans détailler la formulation algébrique (voir Dupray et Moullet, 2004, pour une présentation formelle de ce type de méthode), on peut préciser que la variable expliquée est le salaire en logarithme. Les variables explicatives introduites dans le modèle sont le diplôme de fin d'études (niveau et filière), le temps passé en emploi (hormis celui de l'emploi en cours) et des caractéristiques d'appariement relatives à l'emploi occupé à la date de l'enquête. Ces dernières sont l'ancienneté dans l'entreprise, le secteur public ou privé de l'entreprise, sa taille, sa situation géographique, le type de contrat de travail, le temps de travail. Les valeurs des écarts et des termes de décomposition sont évidemment sensibles aux caractéristiques explicatives retenues dans le modèle (*).

$(*)$ : la robustesse des valeurs des termes de décomposition des écarts de gains est testée au moyen de la méthode du bootstrap (Silber et Weber, 1999). Le principe ici est de répliquer les calculs de décomposition sur 1000 échantillons issus de tirages aléatoires avec remise dans l'ensemble de la population enquêtée. On obtient ainsi une distribution empirique des écarts de gains et de leurs composantes qui permet d'établir des intervalles de confiance.

Tableau 1

\section{Classement des professions selon l'origine - éducative ou liée au marché du travail - de la ségrégation professionnelle qui les affecte}

\begin{tabular}{|c|c|c|c|c|}
\hline & \multicolumn{2}{|c|}{ Cadres et professions intermédiaires } & \multicolumn{2}{|c|}{ Ouvriers et employés } \\
\hline Origine de la ségrégation & Éducative & Liée au marché du travail & Éducative & Liée au marché du travail \\
\hline Professions masculines & $\begin{array}{l}\text { Gl }(£) \\
\text { Ingénieurs (hors } \\
\text { chimie et informatique), } \\
\text { Techniciens de l'industrie, } \\
\text { de la chimie, } \\
\text { de l'agroalimentaire }\end{array}$ & $\begin{array}{l}\text { G2 } \\
\text { Ingénieurs et } \\
\text { techniciens informatiques, } \\
\text { Technico-commerciaux, } \\
\text { Cadres et PI de la } \\
\text { Police, de l'Armée }\end{array}$ & $\begin{array}{l}\text { G6 } \\
O Q \text { et } O N Q \text { de la } \\
\text { métallurgie et de la } \\
\text { mécanique, } \\
O Q \text { et artisans du BTP }\end{array}$ & $\begin{array}{l}\text { G7 } \\
\text { Ouvriers Agricoles, } \\
\text { Chauffeurs et } \bigcirc Q \text { du } \\
\text { transport, } \bigcirc Q \text { et artisans } \\
\text { de l'alimentation, } \\
\text { Employés de police, } \\
\text { de la gendarmerie, } \\
\text { agents de sécurité }\end{array}$ \\
\hline Professions féminines & $\begin{array}{l}\text { G3 } \\
\text { Cadres du para-médical, } \\
\text { Infirmiers, } \\
\text { Assistantes sociales, } \\
\text { éducateurs, Pl de la } \\
\text { banque et des assurances }\end{array}$ & $\begin{array}{l}\text { G4 } \\
\text { Professeurs des écoles, } \\
\text { Conseillers d'éducation, } \\
\text { Pl de la gestion, de } \\
\text { la comptabilité, du } \\
\text { secrétariat et de la } \\
\text { communication }\end{array}$ & \begin{tabular}{l|} 
G8 \\
Aides-soignantes, \\
Coiffeurs et esthéticiennes, \\
Employés de la banque \\
et des assurances
\end{tabular} & $\begin{array}{l}\text { G9 } \\
\text { Secrétaires, } \\
\text { Employés de bureau, } \\
\text { Agents de service } \\
\text { de la Fonction publique, } \\
\text { Serveurs }\end{array}$ \\
\hline Professions mixtes & \multicolumn{2}{|c|}{$\begin{array}{l}\text { G5 } \\
\text { Cadres de la Fonction publique, } \\
\text { Cadres de la banque, des assurances, } \\
\text { PI de la vente, } \\
\text { Techniciens médicaux, } \\
\text { Cadres commerciaux, de la publicité, } \\
\text { Professions de l'information, des Arts \& des spectacles, } \\
\text { Enseignants en collège et lycée, } \\
\text { PI transports, tourisme, } \\
\text { PI commerciales, } \\
\text { Professeurs du supérieur et chercheurs, } \\
\text { Ingénieurs de la chimie. }\end{array}$} & \multicolumn{2}{|c|}{$\begin{array}{l}\text { G10 } \\
\text { Ouvriers du tri, de l'emballage et de l'expédition, } \\
\text { OQ et ONQ textile, } \\
\text { Employés PTT }\end{array}$} \\
\hline
\end{tabular}

(£) G1 pour groupe 1, numéro qui sera repris dans les tableaux et les commentaires relatifs à ce groupe professionnel.

Note : OQ : ouvrier qualifié ; ONQ : ouvrier non qualifié ; PI : profession intermédiaire ; PTT : postes et télécommunications ; BTP : Bâtiment \& travaux publics. 
À partir de ces dix groupes de professions, nous allons maintenant pouvoir énoncer quelques hypothèses sur l'importance relative des écarts de salaire et des composantes de discrimination entre groupes, selon justement la forme de ségrégation et son origine associées à chacun d'eux.

\section{ÉCARTS DE RÉMUNERATION ET DISCRIMINATION SALARIALE : QUELQUES HYPOTHĖSES}

Compte tenu des conditions différentes d'affectation dans les emplois et de la typologie des groupes professionnels réalisée, nous supposons que les écarts de rémunération entre hommes et femmes n'ont pas la même ampleur selon le taux de féminisation et l'origine de la ségrégation du groupe professionnel d'appartenance. Par ailleurs, la discrimination salariale supposée à l'encontre des femmes (partie non justifiée par des différences de caractéristiques individuelles et d'emploi) est susceptible de varier en intensité selon les groupes. Nous pouvons donc avancer quelques hypothèses d'une part sur les valeurs comparées entre groupes des écarts de salaire (H1 à H3), et d'autre part sur les termes de décomposition relatifs à ces écarts (H4 à H6).

Les travaux de Bergmann (1974), et plus récemment ceux de Sorensen (1990), ont souligné la faiblesse des rémunérations des femmes et des hommes dans les professions fortement féminisées. Pour autant, cela n'implique pas que les femmes, par rapport à leurs homologues exerçant la même profession, soient plus pénalisées qu'ailleurs. Il se pourrait au contraire que le nivellement des salaires par le bas permette le rapprochement des conditions de rémunération masculines et féminines (H1).

À l'opposé, une hypothèse concurrente met en avant les propriétés particulières que détiendraient les femmes qui s'insèrent dans des milieux professionnels masculins relativement à leurs consœurs. Les femmes qui exercent des professions masculines (groupes 1,2,6,7) sont sans doute sursélectionnées sur les caractéristiques observées (dont le niveau de diplôme dans les catégories professions intermédiaires et cadres) et sur des attributs inobservés tels que la détermination, la motivation, l'implication dans la vie professionnelle... En effet, si l'arrivée des femmes dans ces professions est freinée, seules celles qui présentent «les plus hautes » qualités observées et inobservées en rapport avec le travail parviendraient à intégrer celles-ci. Les barrières à l'entrée limiteraient alors les différences de situation dans l'emploi. En conséquence, dans les professions masculines, on s'attendrait à une pénalisation salariale des femmes, relativement aux hommes, plus faible qu'ailleurs (H2).

Indépendamment du caractère sexué de la profession, l'origine de la ségrégation influence potentiellement les disparités de salaire entre hommes et femmes et la nature de leurs déterminants. En présence d'une ségrégation professionnelle d'origine éducative sont attendus des écarts de rémunérations selon le sexe plus faibles que lorsque la ségrégation se construit différemment. En effet, dans cette configuration, le diplôme a un rôle prépondérant dans l'affectation des emplois ; ce rôle pourrait perdurer dans la formation des salaires, laissant moins de place à la prise en compte d'autres critères, dont le sexe. Au sein des professions masculines, les groupes 1 et 6 présenteraient, selon cette hypothèse, des écarts de salaire moindres que les groupes 2 et 7 respectivement. Il en serait de même pour les groupes 3 et 8 par rapport aux groupes 4 et 9 dans les professions féminines (H3).

Concernant maintenant l'origine des disparités de salaires, en postulant que les femmes seraient avantagées du point de vue de leurs caractéristiques, notamment scolaires, par rapport aux hommes, dans les professions masculines, on émet l'hypothèse que les différences de gains observées se refléteraient essentiellement, sinon exclusivement, dans des écarts de valorisation monétaire à caractéristiques égales, c'est-à-dire la composante de discrimination (H4).

Par ailleurs, on suppose que dans un milieu masculin les possibilités de paiement différencié des hommes et des femmes (à l'avantage des hommes), à caractéristiques égales, seraient plus fréquentes que dans les professions féminines (H5). En effet, au sein des professions masculines, des capacités non observées et socialement plus facilement reconnues aux hommes telles que la capacité physique, l'aptitude à l'autorité et au commandement, etc. seraient l'objet de différences de rémunération pénalisant les femmes. Dans les professions féminines, les «qualités » féminines non observées seraient moins reconnues en termes de salaire (capacités d'écoute, discrétion, capacité relationnelle...). Consécutivement, les hommes privés de ces qualités seraient moins pénalisés que ne le sont les femmes dans 
les professions masculines sur la base de caractères non observés plutôt perçus comme masculins.

En laissant de côté la composition par sexe des catégories et en s'intéressant à l'origine de la ségrégation, dans la suite de l'hypothèse précédente, il est supposé qu'une ségrégation d'origine éducative atténue le différentiel de valorisation monétaire selon le sexe, des caractéristiques de formation initiale et d'expérience mais également des caractéristiques acquises avec l'affectation dans l'emploi et ce, compte tenu de l'importance probable du diplôme dans le calcul des rémunérations. En résumé, il est supposé une moindre discrimination dans les professions dont l'origine de la ségrégation est principalement éducative (H6).

Avant d'explorer les sources des écarts de rémunération selon les groupes professionnels, examinons dans un premier temps comment se répartissent ces groupes dans la génération 98 et l'importance des écarts de rémunération observés entre hommes et femmes au sein de chacun d'eux.

\section{LES ÉCARTS DE SALAIRES SELON L'ORIGINE DE LA SÉGRÉGATION : LES ESTIMATIONS}

Trois ans après leur sortie du système éducatif en 1998 (voir encadré 2), on compte 41591 jeunes en emploi qui se répartissent inégalement dans les différents groupes de professions présentés plus haut (tableau 2). Si respectivement $41 \%$ et $40 \%$ des jeunes occupent un emploi dans une profession «masculine » et dans une profession «féminine», les professions masculines sont deux fois plus représentées chez les ouvriers et employés que parmi les cadres et professions intermédiaires. La ségrégation apparaît d'ailleurs plus massive au sein des professions de plus faible niveau de qualification que parmi les cadres et professions intermédiaires. Les professions mixtes pèsent en effet pour moitié moins dans les premières que dans les secondes.

\section{Une origine de la ségrégation principalement liée au marché du travail pour les employées et les ouvrières}

Au sein des professions « masculines », celles dont la ségrégation est principalement d'origine éducative (groupes 1 et 6) sont plus importantes en nombre que les autres (groupes 2 et 7). Le constat est le même pour les professions féminines de niveau cadre et profession intermédiaire. À l'inverse, les professions féminines de niveau employé ou ouvrier et caractérisées par une ségrégation qui prend corps sur le marché du travail représentent $19 \%$ de l'ensemble des emplois contre $4,3 \%$ pour les professions féminines dont l'origine de la ségrégation est principalement éducative (groupe 9). Ces constats indiquent que ces dernières catégories professionnelles sont nettement plus soumises que les autres à l'impact de stéréotypes de sexe qui conduisent à renforcer dans l'emploi le clivage sexué des filières éducatives. À titre d'exemple d'une profession appartenant à ce groupe, les secrétaires et assimilés sont non seulement recrutées dans les filières secrétariat-bureautique très féminisées aux niveaux BEP et bac professionnel, voire BTS, mais aussi parmi les sortantes d'autres filières (générales ou tertiaires) comptant un public davantage mixte.

\section{Encadré 2 \\ L'enquête Génération 98}

Les données utilisées appartiennent à l'enquête Génération 98 réalisée par le Centre d'études et de recherches sur les qualifications (Céreq) au printemps 2001. Celle-ci concerne 55000 sortants du système éducatif en 1998, tous niveaux de formation et toutes spécialités de formation confondus, parmi les 750000 primo-sortants cette année-là.

L'enquête rend compte des parcours d'insertion professionnelle: elle fournit des informations individuelles, sociodémographiques et relatives au parcours scolaire, ainsi que des informations sur les différentes séquences d'emploi. Les données, en partie rétrospectives, autorisent ainsi l'analyse des trois premières années de vie active au regard du cursus de formation initiale réalisé (Epiphane et al., 2001). Cette source nous permet en outre de caractériser les jeunes selon leur origine scolaire puis de saisir les différences de situation, notamment salariale, à la date d'enquête. 
Dans chacun des groupes, les différences observées entre le salaire moyen des hommes et celui des femmes, quoique d'ampleurs inégales, sont toujours à l'avantage des premiers. Dans les deux niveaux de qualification distingués, les écarts de rémunération entre hommes et femmes sont les plus élevés au sein des groupes mixtes. Remarquons qu'en dehors des professions mixtes, les catégories cadres et professions intermédiaires sont plus égalitaires en termes de salaires distribués aux hommes et aux femmes que les catégories d'employés et d'ouvriers.

Par ailleurs, chez les cadres et professions intermédiaires, à la fois au sein des professions masculines et au sein des professions féminines, une ségrégation d'origine éducative réduit le différentiel de salaires comparativement à une ségrégation liée au marché du travail. Ceci suggère que, quand le diplôme de formation initiale influence l'allocation des jeunes dans l'emploi, il conditionne également la fixation des rémunérations en limitant les autres sources d'hétérogénéité. De plus, indépendamment de l'origine de la ségrégation, les professions masculines se révèlent en moyenne plus rémunératrices que les professions féminines ; cet avantage est moins saillant parmi les employés et les ouvriers, où les femmes et les hommes du groupe professionnel féminin dont l'origine de la ségrégation est principalement éducative sont les mieux rémunérés. Seul ce groupe fait exception au constat attendu de rémunérations inférieures parmi les professions les plus féminisées. Il est vrai que, si les rémunérations des hommes et des femmes sont différemment affectées par la proportion de femmes dans les professions (Killingsworth, 1990), rien ne permet d'inférer que les écarts de salaire seront systématiquement plus forts dans les professions à taux de féminisation élevé. C'est pourtant bien le cas dans les catégories d'employés et d'ouvriers où les professions féminines affichent le traitement salarial le plus inégalitaire, infirmant l'hypothèse $\mathrm{H} 1$ d'égalisation relative des salaires dans les professions les plus féminisées.

Ces premiers constats, réalisés à partir des données brutes observées, ne tiennent pas compte des situations de formation et d'emploi (niveau de sortie du système éducatif, contrat de travail, temps de travail,

Tableau 2

Quelques caractéristiques des groupes de professions

\begin{tabular}{|c|c|c|c|c|c|c|}
\hline \multicolumn{2}{|c|}{$\begin{array}{c}\text { Groupes professionnels selon l'origine } \\
\text { de la ségrégation... }\end{array}$} & \multirow{2}{*}{\begin{tabular}{|c|}
$\begin{array}{c}\text { Poids relatif } \\
\text { (total = 100) } \\
(\%)\end{array}$ \\
8,1
\end{tabular}} & \multirow{2}{*}{$\begin{array}{c}\begin{array}{c}\text { Part des } \\
\text { femmes } \\
(\%)\end{array} \\
20,0\end{array}$} & \multirow{2}{*}{\begin{tabular}{|c}
$\begin{array}{c}\text { Salaire moyen } \\
\text { des hommes } \\
\text { (euros) }\end{array}$ \\
1473
\end{tabular}} & \multirow{2}{*}{\begin{tabular}{|c|}
$\begin{array}{c}\text { Salaire moyen } \\
\text { des femmes } \\
\text { (euros) }\end{array}$ \\
1423 \\
\end{tabular}} & \multirow{2}{*}{$\begin{array}{c}\begin{array}{c}\text { Écarts de } \\
\text { salaire moyen } \\
\text { (en \%) }\end{array} \\
3,5\end{array}$} \\
\hline Cadres et & 1 -masculine origine éducative & & & & & \\
\hline $\begin{array}{l}\text { professions } \\
\text { intermédiaires }\end{array}$ & $\begin{array}{l}\text { 2-masculine liée au marché } \\
\text { du travail }\end{array}$ & 5,9 & 22,9 & 1722 & 1652 & 4,2 \\
\hline & 3 -féminine origine éducative & 10,6 & 77,7 & 1255 & 1240 & 1,2 \\
\hline & $\begin{array}{l}\text { 4-féminine liée au marché } \\
\text { du travail }\end{array}$ & 6,1 & 78 & 1109 & 1069 & 3,7 \\
\hline & 5 -mixte & 13,4 & 51,3 & 1470 & 1307 & 12,5 \\
\hline \multirow{5}{*}{$\begin{array}{l}\text { Employés et } \\
\text { ouvriers }\end{array}$} & 6-masculine origine éducative & 16,2 & 11,0 & 1074 & 997 & 7,7 \\
\hline & $\begin{array}{l}\text { 7-masculine liée au marché } \\
\text { du travail }\end{array}$ & 10,8 & 16,6 & 1032 & 906 & 13,9 \\
\hline & 8 -féminine origine éducative & 4,3 & 86,4 & 1154 & 1008 & 14,5 \\
\hline & $\begin{array}{l}9 \text {-féminine liée au marché } \\
\text { du travail }\end{array}$ & 19,0 & 77,3 & 985 & 859 & 14,7 \\
\hline & $10-$ mixte & 5,6 & 46,6 & 999 & 849 & 17,7 \\
\hline TOTAL & & 100,0 & 47,4 & 1197 & 1098 & 9,0 \\
\hline
\end{tabular}

Note de lecture : les groupes professionnels de niveau cadre et profession intermédiaire où la ségrégation est principalement d'origine éducative représentent $8,1 \%$ des emplois. Les femmes y représentent $20 \%$ des emplois. Le salaire moyen des hommes y atteint 1473 euros, contre 1423 euros pour les femmes, ce qui représente un écart moyen de 3,5\% à l'avantage des hommes.

Source : Génération 98 - Interrogation 2001. 
taille de l'entreprise...) éventuellement différentes pour les hommes et les femmes, à groupe professionnel donné. Ils doivent être complétés par des analyses contrôlant ces aspects.

\section{Les professions mixtes, plus inégalitaires en termes de salaire}

Les écarts obtenus dans le tableau $\mathbf{3}$, issus de l'estimation économétrique d'une fonction de gains pour chaque groupe professionnel, reviennent à comparer les salaires d'hommes et de femmes qui seraient dotés exactement des mêmes caractéristiques individuelles et d'emploi, calculs permettant d'isoler l'effet salarial propre du sexe, toutes choses égales par ailleurs.

Si l'on retrouve le résultat selon lequel les professions mixtes sont celles où l'effet salarial du sexe est le plus marqué, en revanche l'effet net du sexe s'avère plus ténu dans les professions féminines que masculines et ce, dans les deux grands niveaux de qualification distingués. En d'autres termes, une partie des écarts de rémunération relevés précédemment, notamment dans les professions féminisées des catégories d'employés et d'ouvriers, résulteraient de conditions d'emploi, et éventuellement de diplôme initial, différentes pour les hommes et les femmes au sein de ces professions. À caractéristiques scolaires et d'emplois identiques, les situations salariales des hommes et des femmes seraient donc plus proches dans les professions féminines que masculines, ce qui porterait crédit à l'hypothèse H1.

Dans les professions mixtes, le seul fait d'être un homme (toutes les autres caractéristiques des hommes et des femmes étant identiques) contribue à accroître les salaires de 10 à $12 \%$ selon qu'il s'agit d'employés et d'ouvriers ou de cadres et de professions intermédiaires (tableau 3). C'est dans les professions féminines que le sexe explique le différentiel de gains le plus faible et il est d'autant plus faible que la ségrégation professionnelle est principalement d'origine éducative. Toutes choses égales par ailleurs, être un homme conduit à un supplément de salaire de $6 \%$ chez les employés et ouvriers des professions féminines où la ségrégation est d'origine éducative, le gain salarial étant d'environ $3 \%$ pour le groupe professionnel équivalent chez les cadres et professions intermédiaires (tableau 3).

Ces mesures de l'impact salarial de la variable sexe montrent des effets différenciés - du simple au triple -

Tableau 3

Impact salarial du sexe selon les groupes professionnels et la catégorie sociale

\begin{tabular}{|l|l|c|}
\hline \multicolumn{2}{|c|}{ Groupes professionnels selon l'origine de la ségrégation... } & $\begin{array}{c}\text { Écart de salaires estimé entre } \\
\text { hommes et femmes } \\
\text { (en \%) }\end{array}$ \\
\hline Cadres et professions intermédiaires & 1 -masculine origine éducative & $9,3\left(^{*}\right)$ \\
\cline { 2 - 3 } & 2 -masculine liée au marché du travail & 8,5 \\
\cline { 2 - 3 } & 3-féminine origine éducative & 3,3 \\
\cline { 2 - 3 } & 4 -féminine liée au marché du travail & 4,9 \\
\cline { 2 - 3 } & 5 -mixte & 11,7 \\
\hline Employés et ouvriers & 6 -masculine origine éducative & 7,1 \\
\cline { 2 - 3 } & 7 -masculine liée au marché du travail & 10,1 \\
\cline { 2 - 3 } & 8 -féminine origine éducative & 6,1 \\
\cline { 2 - 3 } & 9 -féminine liée au marché du travail & 7,6 \\
\cline { 2 - 3 } & 10 -mixte & 10,0 \\
\hline
\end{tabular}

Notes de lecture :

(*) dans le groupe 1 composé des professions de cadres et intermédiaires, masculines et où l'origine de la ségrégation est principalement d'origine éducative, le fait d'être un homme accroît le salaire obtenu après trois ans de vie active de $9,3 \%$, toutes choses égales par ailleurs. Il s'agit du paramètre de la variable croisant le sexe et le groupe professionnel d'appartenance obtenu par l'estimation d'une fonction de gains comportant également les variables explicatives suivantes : ancienneté dans l'entreprise, taille et localisation géographique de l'entreprise, temps et contrat de travail, expérience professionnelle préalable au dernier emploi, niveau et spécialité de formation.

Source : Génération 98 - Interrogation 2001. 
selon l'appartenance professionnelle. Cependant, elles livrent une photographie encore trompeuse de la réalité car, à groupe professionnel donné, si les hommes et les femmes ne possèdent pas en moyenne les mêmes caractéristiques, ils ne les valorisent pas non plus forcément de la même façon. L'intérêt de prendre en compte ces deux aspects dans le calcul de l'écart de rémunération entre hommes et femmes réside dans la possibilité de décomposer cet écart en deux parties afin d'isoler une partie non justifiable (écart de valorisation monétaire des caractéristiques) d'une partie - économiquement - justifiable (différences de distribution des caractéristiques individuelles et d'emploi; voir encadré 1). Pour réaliser cela, il est nécessaire d'opérer en deux temps. Il faut d'abord calculer, séparément pour les hommes et pour les femmes, la valorisation monétaire des différentes caractéristiques individuelles et d'emploi. Il faut ensuite tenir compte des différences de distribution de ces caractéristiques existant entre hommes et femmes en appliquant ces valorisations monétaires aux carac- téristiques correspondant, pour chaque sexe, à leur situation moyenne au sein de la profession étudiée. Nous aboutissons alors aux résultats du tableau 4.

Il apparaît alors que les écarts salariaux sont plus importants au sein des professions féminines qu'au sein des professions masculines, parmi les employés et ouvriers ; cela tend à infirmer l'idée que la densité de maind'œuvre féminine contribue à l'homogénéisation des conditions de rémunération selon le sexe. A contrario, cette hypothèse (H1) se vérifie dans les catégories cadres et professions intermédiaires où le groupe professionnel féminin, dont l'origine de la ségrégation est principalement d'origine éducative, enregistre le retard salarial le plus faible (1,9\% cf. tableau 4, colonne $\mathbf{1})$.

Les professions mixtes présentent, comme précédemment, les écarts de salaire les plus importants pour les deux niveaux de qualification. Ces professions sont celles pour lesquelles les deux origines de la ségrégation sont faibles ou se compensent. Ainsi, parmi les cadres et professions intermédiaires, les hommes ont

Tableau 4

Résultats de la modélisation des différences de salaire entre hommes et femmes selon le groupe professionnel et la catégorie sociale (en \%)

\begin{tabular}{|c|c|c|c|c|}
\hline \multicolumn{2}{|c|}{$\begin{array}{c}\text { Groupes professionnels selon l'origine } \\
\text { de la ségrégation... }\end{array}$} & \multirow{2}{*}{$\begin{array}{l}\begin{array}{l}\text { Différences } \\
\text { estimées } \\
\text { de salaire }\end{array} \\
3,6 * *\end{array}$} & \multirow{2}{*}{$\begin{array}{l}\text { imputables à des } \\
\text { différences de } \\
\text { caractéristiques (*) } \\
4,2\end{array}$} & \multirow{2}{*}{$\begin{array}{c}\text { imputables à des } \\
\text { différences de } \\
\text { valorisation }\end{array}$} \\
\hline Cadres et & 1 -masculine origine éducative & & & \\
\hline professions & 2 -masculine liée au marché du travail & 5,7 & 1,6 & 7,4 \\
\hline & 3 - féminine origine éducative & 1,9 & 0,4 & 1,5 \\
\hline & 4 -féminine liée au marché du travail & 4,0 & 0,4 & 3,6 \\
\hline & 5 -mixte & 12,4 & 0,9 & 11,5 \\
\hline \multirow{5}{*}{$\begin{array}{l}\text { Employés et } \\
\text { ouvriers }\end{array}$} & 6 -masculine origine éducative & 6,9 & 0,9 & 5,9 \\
\hline & 7-masculine liée au marché du travail & 13,8 & 4,8 & 8,6 \\
\hline & 8 -féminine origine éducative & 14,7 & $10,3 * * *$ & $4,0 * * *$ \\
\hline & 9 -féminine liée au marché du travail & 15,6 & 8,1 & 6,9 \\
\hline & $10-$ mixte & 17,1 & 6,5 & 9,9 \\
\hline
\end{tabular}

(*) le niveau d'éducation, la spécialité de la filière, l'expérience professionnelle nette du temps passé dans l'emploi occupé, l'ancienneté dans cet emploi, le statut et le temps de travail, la taille et la localisation géographique, le secteur public ou privé de l'établissement.

Notes de lecture : on évalue ici, pour chaque groupe professionnel, les rémunérations pour des hommes et des femmes dont les caractéristiques correspondent pour chaque sexe à leur situation moyenne au sein de la profession étudiée.

(**) Dans le groupe 1, composé des professions intermédiaires et cadres, et masculines, où l'origine de la ségrégation est principalement éducative, les hommes gagnent $3,6 \%$ de plus que les femmes.

(***) Dans le groupe 8 composé de professions féminines d'employés ou d'ouvriers où la ségrégation est principalement d'origine éducative, les hommes gagnent $10,3 \%$ de plus que les femmes du fait de caractéristiques individuelles et d'emploi plus avantageuses que celles de leurs consœurs, et $4 \%$ de plus, compte tenu d'un avantage dans la valorisation monétaire de leurs caractéristiques comparativement aux femmes possédant des caractéristiques identiques. Au total, ils gagnent 14,7 \% de plus que les femmes ; 1,147 étant le produit de 1,103 et 1,04.

Source : Génération 98 - Interrogation 2001. 
des salaires $12 \%$ supérieurs en moyenne à leurs consœurs, cet écart atteignant $17 \%$ chez les employés et ouvriers.

À composition sexuée des professions donnée, les écarts de rémunération sont les plus élevés lorsque la ségrégation professionnelle liée au marché du travail joue un rôle important. Ce résultat vaut pour les deux niveaux de qualification. De ce point de vue, chez les employés et ouvriers, c'est au sein de professions masculines que l'écart est le plus important : près de $14 \%$ dans le groupe 7 contre $7 \%$ dans le groupe 6 , où la ségrégation est principalement d'origine éducative. Au total, l'hypothèse $\mathrm{H} 3$ d'un moindre écart de gains entre hommes et femmes dans les situations où la ségrégation professionnelle est essentiellement d'origine éducative est validée.

Pour résumer, la ségrégation des professions par sexe semble décroître au fur et à mesure que le niveau de qualification des emplois s'élève. En outre, malgré les niveaux de revenus modestes des employés et des ouvriers $^{3}$, les écarts de rémunération entre hommes et femmes y sont en moyenne plus importants que parmi les cadres et professions intermédiaires.

Les professions mixtes se révèlent les plus inégalitaires en termes de salaires, que cela soit parmi la catégorie des cadres et professions intermédiaires ou parmi celle des employés et ouvriers. Pour cette dernière catégorie, les professions féminisées sont plus inégalitaires que les professions "masculines» alors que l'inverse est vrai dans les niveaux de qualification supérieurs. Enfin, dans tous les cas, une ségrégation dont l'origine est principalement éducative s'accompagne d'une réduction du différentiel de salaires entre les sexes par rapport à celui observé dans les professions dont la ségrégation ressort principalement de mécanismes d'affectation des individus aux emplois.

Nous recherchons maintenant l'origine des écarts de salaires selon les groupes en les décomposant entre ce qui relève de différences de caractéristiques individuelles et d'emploi selon le sexe et ce qui ressort de valorisations monétaires différenciées de celles-ci dès lors qu'elles sont identiques (deux dernières colonnes du tableau 4 ).

\footnotetext{
${ }^{3}$ Dont on peut penser qu'ils devraient niveler les écarts selon le sexe.
}

\section{Dans les professions masculines supérieures, le désavantage salarial des femmes relève de la discrimination}

$\mathrm{Au}$ sein des professions masculines de catégories cadres et professions intermédiaires, à la différence des autres groupes professionnels, les écarts de caractéristiques individuelles et d'emploi sont en moyenne à l'avantage des femmes. En l'occurrence, elles possèdent une ancienneté dans leur entreprise plus élevée que celle des hommes, travaillent en plus grande proportion dans les grandes entreprises et plus souvent à Paris qu'en province, comparativement à leurs homologues masculins. Elles sont surtout, pour deux tiers d'entre elles, titulaires de diplômes de niveau 1 ou 2. Autrement dit, si hommes et femmes étaient rémunérés de la même façon, alors les premiers gagneraient de 1,6 à $4,2 \%$ de moins que les secondes (colonne 2 chiffrée du tableau 4). Comme tel n'est pas le cas, cela signifie que l'on ne rémunère pas de la même manière des caractéristiques identiques pour les hommes et pour les femmes. Ainsi, la composante de discrimination de l'écart de gains à l'avantage des hommes, allant de 7 à $8 \%$, fait plus que contrebalancer les différences de caractéristiques individuelles. De ce fait, les modestes écarts salariaux entre hommes et femmes constatés dans ces groupes (entre 4 et $6 \%$ ) masquent une discrimination à l'encontre des femmes de plus forte ampleur. Ces résultats confortent l'hypothèse $\mathrm{H} 4$ selon laquelle, au sein des professions masculines, les femmes seraient en moyenne mieux payées que leurs homologues masculins en l'absence de discrimination salariale.

Chez les cadres et professions intermédiaires, la composante inexpliquée des écarts de salaires s'avère largement prédominante, quel que soit le groupe professionnel. Toutefois, pour les groupes féminins et mixtes, les écarts de rémunération liés aux différences de caractéristiques individuelles et d'emploi, légèrement en faveur des hommes, s'ajoutent à ceux issus des différences de valorisation monétaire à l'encontre des femmes.

Parmi les employés et les ouvriers, les écarts de caractéristiques à l'avantage des hommes sont les plus importants dans les professions féminines, notamment dans le groupe 8 dont la ségrégation est principalement d'origine éducative. Ces écarts portent principalement sur le taux de travail à temps 
Tableau 5

Part des salariés à temps partiel selon le sexe et le groupe professionnel (en \%)

\begin{tabular}{|l|l|c|c|}
\hline \multicolumn{2}{|c|}{ Groupes professionnels selon l'origine de la ségrégation... } & Hommes & Femmes \\
\hline \multirow{4}{*}{$\begin{array}{l}\text { Cadres et professions } \\
\text { intermédiaires }\end{array}$} & 1 -masculine origine éducative & 1,6 & 5,4 \\
\cline { 2 - 4 } & 2-masculine liée au marché du travail & 1,4 & 2,6 \\
\cline { 2 - 4 } & 3 -féminine origine éducative & 8,8 & 15,5 \\
\cline { 2 - 4 } & 4 -féminine liée au marché du travail & 7,2 & 8,1 \\
\cline { 2 - 4 } & 5 -mixte & 2,7 & 10,4 \\
\hline Employés et ouvriers & 6-masculine origine éducative & 7,0 & 15,7 \\
\cline { 2 - 4 } & 7-masculine liée au marché du travail & 4,2 & 17,6 \\
\cline { 2 - 4 } & 8-féminine origine éducative & 12,9 & 30,3 \\
\cline { 2 - 4 } & 9-féminine liée au marché du travail & 7,4 & 22,9 \\
\cline { 2 - 4 } & 10 -mixte & & 0.7 \\
\hline
\end{tabular}

Note de lecture : $1,6 \%$ des hommes appartenant aux groupes professionnels de niveau cadre ou profession intermédiaire et dont la ségrégation est principalement d'origine éducative travaillent à temps partiel.

Source : Génération 98 - Interrogation 2001.

partiel, bien plus élevé chez les femmes que chez les hommes (tableau 5) et sur la plus forte proportion des femmes issues des filières tertiaires de formation, au détriment des filières générales et industrielles plus empruntées par les hommes et qui offrent les meilleures valorisations salariales. De plus, comme chez les cadres et professions intermédiaires, le poids de la discrimination est plus important dans les professions masculines que dans les groupes féminins ; ceci conforte l'idée que les aptitudes observées et non observées exigées dans ces professions laissent potentiellement plus de marge à des pratiques discriminatoires ou des pratiques dont les fondements sont moins objectivables (H5).

\section{Une amplitude maximale de la discrimination dans les groupes professionnels mixtes}

Pour ce qui est de la composante de l'écart de rémunération imputable à des différences de valorisation monétaire des caractéristiques individuelles et d'emploi, le handicap salarial des femmes est le plus marqué pour les groupes mixtes 5 et 10 (colonne 3 du tableau 4). Ce résultat peut surprendre, dans la mesure où, dans ces professions, l'équilibre relatif entre main-d'œuvre masculine et main-d'œuvre féminine laisse présumer que les femmes sont mieux en mesure de défendre leurs conditions de rémunération-comparativement aux femmes au sein des professions masculines en particulier. Il confirme aussi un paradoxe déjà souligné par Couppié et Epiphane (2004) qui pointent que mixité n'est pas caution d'équité. Ce constat peut trouver sa source dans la nature des professions du groupe mixte aux niveaux cadres et professions intermédiaires. Il concentre en effet des professions intermédiaires commerciales dont on sait qu'une partie significative de la rémunération varie selon les résultats du salarié. Ainsi ces situations se démarquent de celles des autres salariés par des pratiques d'individualisation de la négociation salariale plus développées, pratiques susceptibles d'élargir l'éventail des critères sur la base desquels est calculée la rémunération. Cette plus forte individualisation des salaires dans ces professions conduit à une élévation de la part non expliquée des écarts par le modèle, sans que l'on puisse l'imputer forcément à une véritable pratique discriminatoire.

Quant au groupe mixte des catégories professionnelles d'employés et d'ouvriers (groupe 10), il rassemble principalement les ouvriers du tri et de l'emballage, d'une part, et les ouvriers du nettoyage, d'autre part. Or, dans les différentes professions étudiées, il existe une variance intra-catégorielle des emplois selon le sexe que le niveau d'agrégation des nomenclatures utilisées ne permet pas de saisir. Ainsi, 
compte tenu de la pénibilité invoquée des tâches dans les métiers liés à la logistique et à l'expédition, il est traditionnellement observé une segmentation des postes occupés par les hommes et femmes, réservant les moins pénibles physiquement à ces dernières. De ce fait, en raison de différences compensatrices, les hommes seraient davantage rémunérés que les femmes compte tenu des conditions de travail plus fatigantes (Macpherson et Hirsh, 1995). Dans le secteur du nettoyage, les jeunes hommes, entrés à la suite de la mise en place de filières de formation spécifiques, ont probablement davantage profité des transformations organisationnelles et technologiques $\mathrm{du}$ secteur que les jeunes femmes, plus souvent cantonnées aux formes traditionnelles de l'activité (Djellal, 2002).

$\mathrm{Du}$ point de vue de l'origine de la ségrégation, la ségrégation de source principalement éducative atténue partout l'ampleur de la discrimination à l'encontre des femmes, à l'exception des professions masculines intermédiaires et de l'encadrement, tendant à confirmer l'hypothèse H6. Au total, l'origine de la ségrégation professionnelle comme le degré de mixité de la profession - du masculin au féminin - influencent l'ampleur de la discrimination salariale.

Le poids du temps partiel parmi les femmes de niveaux employé et ouvrier explique en partie l'importance des écarts de rémunération établis au tableau 4. On sait en effet que les différences de durée du travail entre hommes et femmes jouent un rôle important dans leur différentiel de salaires (Meurs et Ponthieux, 2000). En se focalisant uniquement sur les salariés à temps complet, les différentiels de salaires se réduisent, notamment dans les groupes professionnels où l'on compte un nombre important

\section{Tableau 6
Écarts de salaire selon le sexe pour les salariés à temps plein}

\begin{tabular}{|c|c|c|c|c|}
\hline \multicolumn{2}{|c|}{ Groupes professionnels selon l'origine de la ségrégation... } & \multirow{2}{*}{$\begin{array}{c}\text { Différence } \\
\text { estimée de } \\
\text { salaire } \\
1,7 \% \%^{\star *}\end{array}$} & \multirow{2}{*}{$\begin{array}{c}\text { imputable à des } \\
\text { différences de } \\
\text { caractéristiques } \\
\left({ }^{*}\right) \\
-6,0 \%\end{array}$} & \multirow{2}{*}{$\begin{array}{c}\text { imputable à des } \\
\text { différences de } \\
\text { valorisation }\end{array}$} \\
\hline Cadres et & 1- masculine origine éducative & & & \\
\hline professions & 2- masculine liée au marché du travail & $5,5 \%$ & $-2,2 \%$ & $7,8 \%$ \\
\hline & 3-féminine origine éducative & $-0,9 \%$ & $-2,4 \%$ & $1,5 \%$ \\
\hline & 4-féminine liée au marché du travail & $3,8 \%$ & $-0,3 \%$ & $4,1 \%$ \\
\hline & 5-mixte & $9,4 \%$ & $-1,8 \%$ & $11,4 \%$ \\
\hline \multirow{5}{*}{$\begin{array}{l}\text { Employés et } \\
\text { ouvriers }\end{array}$} & 6- masculine origine éducative & $5,6 \%$ & $-0,2 \%$ & $5,4 \%$ \\
\hline & 7-masculine liée au marché du travail & $9,3 \%$ & $2,2 \%$ & $7,0 \%$ \\
\hline & 8 -féminine origine éducative & $10,5 \%$ & $6,0 \% * * *$ & $4,3 \% * * *$ \\
\hline & 9-féminine liée au marché du travail & $8,5 \%$ & $1,1 \%$ & $7,2 \%$ \\
\hline & 10-mixte & $8,7 \%$ & $1,1 \%$ & $7,6 \%$ \\
\hline
\end{tabular}

(*) le niveau d'éducation, la spécialité de la filière, l'expérience professionnelle nette du temps passé dans l'emploi occupé, l'ancienneté dans cet emploi, le statut et le temps de travail, la taille et la localisation géographique, le secteur public ou privé de l'établissement.

Notes de lecture :

On évalue ici, pour chaque groupe professionnel, les rémunérations pour des hommes et des femmes travaillant à temps plein dont les caractéristiques correspondent pour chaque sexe à leur situation moyenne au sein de la profession étudiée.

(**) Dans le groupe 1, composé des professions intermédiaires et cadres et masculines, où l'origine de la ségrégation est principalement éducative, les hommes gagnent $1,7 \%$ de plus que les femmes.

(***) Dans le groupe 8 composé de professions féminines d'employés ou d'ouvriers où la ségrégation est principalement d'origine éducative, les hommes gagnent $6 \%$ de plus que les femmes du fait de caractéristiques individuelles et d'emploi plus avantageuses que celles de leurs consœurs, et $4,3 \%$ de plus, compte tenu d'un avantage dans la valorisation monétaire de leurs caractéristiques, comparativement aux femmes possédant des caractéristiques identiques. Au total, ils gagnent $10,5 \%$ de plus que les femmes ; $10,5 \%$ étant le résultat du produit de $6,0 \%$ et $4,3 \%$.

Source : Génération 98 - Interrogation 2001. 
de femmes travaillant à temps partiel ou pour les groupes dont le différentiel de travail à temps partiel entre hommes et femmes est élevé, comme dans le groupe 7 (tableaux 5 et 6). Globalement, la part injustifiée des écarts de rémunérations ne change pas en proportion quel que soit le groupe professionnel, ce qui indique que, dans l'ensemble, le traitement salarial des caractéristiques individuelles et d'emploi des hommes et des femmes ne varie pas significativement, qu'ils travaillent à temps complet ou à temps partiel. En revanche, la part liée aux différences de caractéristiques entre hommes et femmes évolue légèrement, notamment aux niveaux cadres et professions intermédiaires, et ce au détriment des hommes (tableau 6). Ainsi, en l'absence de pratiques discriminatoires, aux niveaux cadres et professions intermédiaires, les femmes travaillant à temps complet gagneraient systématiquement plus que leurs homologues masculins.

\section{UNE DISCRIMINATION SALARIALE PORTÉE PAR LES CARACTÉRISTIQUES DE L’EMPLOI OCCUPÉ}

Une forme de décomposition plus approfondie consiste à désagréger les caractéristiques individuelles et d'emploi et les composantes de valorisation monétaire entre ce qui relève de l'appariement de l'individu à l'emploi, lequel conjugue à la fois des souhaits individuels et les besoins des entreprises, et ce qui renvoie plus pleinement à des choix et préférences individuelles antérieurs à la prise d'emploi. En d'autres termes, dans le premier cas, il s'agit de l'ensemble des paramètres qui concourent à définir la situation d'emploi (secteur d'activité, contrat et temps de travail, taille de l'entreprise, temps passé dans l'entreprise, localisation géographique). Dans le second cas, il s'agit des caractéristiques d'offre de travail acquises avant l'entrée sur le marché du travail (niveau de formation, spécialité) ou dont on peut supposer qu'elles ne sont pas contingentes à la situation singulière de l'individu dans l'emploi occupé à l'enquête - qui fournit le salaire -, à l'exemple de la durée de l'expérience professionnelle acquise avant l'arrivée dans l'emploi. La dénomination de « caractéristiques d'offre de travail » nous servira à désigner ce deuxième ensemble de dimensions.

La décomposition entre caractéristiques d'appariement et d'offre de travail permet de préciser dans quelles conditions s'exerce la discrimination salariale (tableau 7). Compte tenu de la force du lien entre diplôme et affectation d'emploi dans les professions des groupes 1, 3, 6 et 8 (dont la ségrégation est principalement d'origine éducative), on s'attend en particulier à ce que la discrimination portant sur les attributs d'offre de travail soit plus faible dans ces groupes (H7).

Sur la base des écarts de salaire entre hommes et femmes résultant exclusivement des différences d'offre de travail, les femmes dans les professions intermédiaires et de l'encadrement sont mieux loties que les hommes au regard notamment de leur capital scolaire (les chiffres négatifs dans la troisième colonne du tableau 7 indiquent un avantage salarial pour les femmes). Dans les catégories d'employés et d'ouvriers, seuls les groupes de professions masculines voient les femmes plus avantagées que leurs confrères au regard de leurs caractéristiques d'offre de travail. À l'opposé, et à l'exception des professions masculines intermédiaires et supérieures dont l'origine de la ségrégation prend corps sur le marché du travail, les hommes bénéficient de conditions d'appariement à l'emploi meilleures que celles de leurs homologues féminines (première colonne du tableau 7); dès lors, les écarts de salaire issus des différences d'attributs individuels et d'emploi entre hommes et femmes portent quasi exclusivement sur les caractéristiques d'appariement. Si, par exemple, les emplois de cadre poursuivent leur féminisation avec les nouvelles générations, les disparités de conditions d'emploi entre hommes et femmes persistent. Les emplois de cadres sont en effet polarisés en deux filières, relationnelle pour les femmes, plus technique et d'encadrement pour les hommes (Okba, 2004 ; Pochic, 2005).

Du point de vue des composantes inexpliquées de l'écart salarial, assimilables à la discrimination, sont principalement en cause les conditions d'emploi qui pénalisent quasi systématiquement les femmes, à l'exception des professions féminines de catégories employés-ouvriers du groupe 8 . Cependant, dans ces dernières professions, les femmes sont clairement défavorisées au regard de la valorisation monétaire de leurs caractéristiques d'offre de travail, puisque si on s'en tient à ces attributs, elles percevraient des rémunérations près de $12 \%$ inférieures à celles de leurs homologues masculins. 
Tableau 7

\section{Décomposition des écarts de salaire entre hommes et femmes selon les caractéristiques $d^{\prime}$ 'offre de travail et d'appariement (en \%)}

\begin{tabular}{|c|c|c|c|c|c|}
\hline \multirow{3}{*}{\multicolumn{2}{|c|}{$\begin{array}{l}\text { Groupes professionnels selon } \\
\text { l'origine de la ségrégation... }\end{array}$}} & \multicolumn{4}{|c|}{ Composantes des écarts de salaires ... } \\
\hline & & \multicolumn{2}{|c|}{ Caractéristiques d'appariement } & \multicolumn{2}{|c|}{ Caractéristiques d'offre de travail } \\
\hline & & $\begin{array}{c}\frac{\text { Disparité }}{\text { Imputable }} \\
\text { à des } \\
\text { différences de } \\
\text { caractéristiques }\end{array}$ & $\begin{array}{l}\frac{\text { Discrimination }}{\text { Imputable à }} \\
\text { des différences } \\
\text { de valorisation }\end{array}$ & $\begin{array}{c}\text { Disparité } \\
\text { Imputable } \\
\text { à des } \\
\text { différences de } \\
\text { caractéristiques }\end{array}$ & $\begin{array}{l}\frac{\text { Discrimination }}{\text { Imputable à }} \\
\text { des différences } \\
\text { de valorisation }\end{array}$ \\
\hline \multirow{5}{*}{$\begin{array}{l}\text { Cadres et } \\
\text { professions } \\
\text { intermédiaires }\end{array}$} & 1 - masculine origine éducative & 1,1 & 15,2 & $-5,2$ & $-6,1$ \\
\hline & $\begin{array}{l}\text { 2- masculine liée au marché du } \\
\text { travail }(£)\end{array}$ & $-0,4^{*}$ & $38,4^{* *}$ & $-1,1 *$ & $-22,5^{\star \star}$ \\
\hline & 3-féminine origine éducative & 1,6 & 18,0 & $-1,1$ & $-14,0$ \\
\hline & 4-féminine liée au marché du travail & 0,9 & 13,8 & $-0,4$ & $-9,0$ \\
\hline & 5-mixte & 2,1 & 10,2 & $-1,2$ & 1,1 \\
\hline \multirow{5}{*}{$\begin{array}{l}\text { Employés et } \\
\text { ouvriers }\end{array}$} & 6-masculine origine éducative & 1,7 & 4,3 & $-0,8$ & 1,6 \\
\hline & 7- masculine liée au marché du travail & 5,4 & 11,4 & $-0,6$ & $-2,5$ \\
\hline & 8 -féminine origine éducative & 6,0 & $-6,8$ & 4,1 & 11,6 \\
\hline & 9-féminine liée au marché du travail & 7,4 & 8,7 & 0,6 & $-1,7$ \\
\hline & 10- mixte & 5,7 & 4,6 & 0,7 & 5,1 \\
\hline
\end{tabular}

Notes de lecture :

(£) Par commodité, lorsque l'origine de la ségrégation est aussi ou principalement liée au marché du travail, on met cet aspect en avant.

(*) Dans le groupe 2, les hommes gagnent 0,4\% de moins que les femmes du fait des différences dans les caractéristiques d'emploi et $1,1 \%$ de moins du fait des différences dans les caractéristiques d'offre de travail.

(**) Toujours dans le groupe 2, les hommes gagnent 38,4\% de plus, compte tenu d'un avantage dans la valorisation monétaire de leurs caractéristiques d'emploi comparativement aux femmes possédant des caractéristiques identiques et $22,5 \%$ de moins du fait de la valorisation monétaire différente de leurs caractéristiques d'offre de travail. Au total, dans ce groupe, les hommes gagnent : $(1-0.004)(1+0.384)(1-0.011)(1-0.225)$ $=5,7 \%$ ( $c f$. tableau 4) de plus que leurs consœurs du même groupe professionnel.

Source : Génération 98 - Interrogation 2001.

Au total, l'avantage que les femmes, relativement aux hommes, sont parfois en mesure de tirer de la valorisation monétaire de leurs diplômes et expérience professionnelle est surcompensé par le désavantage qu'elles subissent en matière de reconnaissance salariale de leurs conditions d'emploi (contrat de travail, temps de travail,...).

Globalement, dans les catégories cadres et professions intermédiaires, excepté le cas des professions mixtes, les femmes voient leurs attributs d'offre de travail mieux reconnus au niveau salarial que ceux des hommes (colonne 4 du tableau 7). C'est particulièrement le cas dans les professions d'ingénieurs et techniciens de l'informatique qui représentent une bonne part de l'effectif du groupe 2, pour lesquelles les hommes percevraient virtuellement environ $22 \%$ de moins que leurs consœurs. Cela étant, ils récupèrent leur avantage par le biais de conditions d'appariement à l'emploi qui leur assurent virtuellement un revenu moyen $38 \%$ supérieur à celui de leurs homologues féminins (colonne 2).

Parmi les ouvriers et employés, la rétribution des attributs d'offre de travail se révèle en moyenne plus élevée pour les hommes dans trois cas sur cinq, et notamment dans les professions masculines ou féminines dont la ségrégation est principalement d'origine éducative. Une ségrégation d'origine éducative n'assure donc en rien que les attributs scolaires des femmes seront rémunérés de façon voisine ou à l'égal de ceux des hommes, infirmant l'hypothèse $\mathrm{H} 7$.

De ce fait, pour la catégorie des employés et des ouvriers, si l'origine principalement éducative de la 
ségrégation tend à réduire la discrimination (H6), aussi bien dans les professions masculines que féminines, c'est presque exclusivement dans la reconnaissance des caractéristiques d'appariement que se réalise l'ajustement.

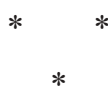

La mesure des écarts de salaire entre hommes et femmes et de leurs compositions ont permis de mettre en lumière des différences de situations notables entre professions regroupées selon leur degré de ségrégation et la construction de celle-ci - ségrégation d'origine éducative ou professionnelle.

Pour ce qui est du positionnement salarial des femmes relativement aux hommes, celles-ci sont les mieux placées dans les professions féminines au sein des catégories de cadres et de professions intermédiaires. Leur rémunération est en effet la plus proche de celles des hommes dans les professions féminines à ségrégation principalement d'origine éducative (2\%).

L'identification des sources des écarts salariaux entre ce qui relève des caractéristiques individuelles et d'emploi et ce qui renvoie à de la discrimination révèle que les femmes ne sont pas forcément les moins discriminées dans les professions où elles accusent un retard salarial assez faible, à l'exemple des professions masculines de niveaux cadres et professions intermédiaires. Ce sont les seuls groupes pour lesquels les caractéristiques individuelles d'offre de travail et d'emploi des femmes sont plus importantes que celles des hommes. Consécutivement, si les femmes étaient rémunérées à l'égal des hommes (même valorisation monétaire des attributs individuels et d'emploi), elles gagneraient en moyenne plus que ces derniers, respectivement $4,2 \%$ et $1,6 \%$ dans les groupes masculins de cadres et professions intermédiaires. C'est donc parce qu'elles sont discriminées que leur salaire est peu éloigné de celui des hommes. La discrimination est d'ailleurs en moyenne plus forte dans les professions masculines que féminines, et ce pour les deux niveaux de qualification considérés (cadres et professions intermédiaires d'une part et employés et ouvriers d'autre part). Elle est toutefois encore plus prononcée dans les groupes professionnels mixtes.

En termes de politiques publiques, ces constats suggèrent que soit accordée une attention particu- lière aux professions masculines et mixtes où les femmes ont à subir un traitement salarial très désavantageux. Il faudrait bien sûr cerner un plus grand nombre de paramètres relatifs aux situations d'emplois, notamment une catégorisation des professions et niveaux de qualification plus fine, pour améliorer encore la comparabilité des situations. Par ailleurs, on peut se demander si une partie des différences de valorisation monétaire ne résulte pas de différences sexuées des mécanismes de salaires compensateurs de désutilités professionnelles, tel qu'évoqués par Macpherson et Hirsh (1995). Dans ce cadre, soient les désutilités associées aux conditions de travail apparaissent plus élevées pour les hommes que pour les femmes - nécessitant des compensations supérieures -, soit le mécanisme de compensation apparaît en lui-même moins efficace pour les femmes que pour les hommes - par exemple en négligeant plus facilement la reconnaissance, dans les grilles de classification et de salaire, de qualités statistiquement et socialement attribuées aux femmes (l'écoute, l'attention aux autres, etc.) ou de compétences acquises dans la sphère domestique (soins à la personne, etc.) (Lemière et Silvera, 2001). Enfin, rien ne dit que ces différences de valorisation monétaire au détriment des femmes ne ressortent pas également pour partie d'arbitrages féminins en faveur d'autres attributs de l'emploi. Pour certaines d'entre elles, l'importance du salaire serait plus fortement contrebalancée par d'autres critères tels qu'une plus grande maîtrise de leur temps de travail ou une préférence pour un milieu de travail moins stressant par rapport à des emplois où le rythme de travail est soumis à de fortes pressions (Dupray, 2005). De ce point de vue, la composante non expliquée des écarts de gains représente probablement une limite haute de la discrimination.

Par ailleurs, il apparaît qu'à degré de mixité donné, les professions dont la ségrégation est d'origine éducative sont porteuses des moins mauvaises conditions de rémunération pour les femmes (par rapport aux hommes) et présentent, à l'exception des professions masculines supérieures et intermédiaires, une moindre discrimination. À cet égard, cette origine éducative de la ségrégation semble assurer un meilleur emboîtement entre les savoirs acquis en formation initiale et les compétences qui seront reconnues d'un point de vue salarial, à l'exemple des professions du secteur médical et para-médical. 
De plus, pour tous les groupes professionnels, la discrimination salariale à l'encontre des femmes est largement due aux caractéristiques de l'emploi occupé (conditions d'appariement). À conditions identiques, par exemple en $\mathrm{CDD}$, en province dans une PME et avec un an d'ancienneté, elles percevront un salaire inférieur à celui de leurs homologues dans la même situation. En effet, les caractéristiques d'offre de travail sont légèrement mieux rémunérées pour les femmes dans la majorité des groupes professionnels, notamment au sein des catégories de cadres et de professions intermédiaires. Cet avantage en leur faveur sur les conditions de formation initiale et d'expérience professionnelle reste toutefois insuffisant pour compenser les différences de valorisation moné- taire à l'avantage des hommes relatives aux conditions d'emploi.

À cet égard, on peut conclure que la discrimination salariale des femmes se construit véritablement dans l'emploi avec toutes les limites soulignées précédemment. La prime au diplôme enregistrée par les femmes cadres et dans les professions intermédiaires, c'est-à-dire l'avantage qu'elles sont en mesure de tirer de la valorisation de leurs diplôme en matière de rémunération, est effacée par «les pénalités » relatives qu'elles supportent par la suite en matière de rémunération de leurs conditions d'emploi. Ce traitement en leur défaveur semble partagé en intensité, sinon en usage, par l'ensemble des professions.

\section{Bibliographie}

Altonji J.G., Blank R.M. (1999), "Race and Gender in the labor market", in Ashenfelter, O. Et Card, D. (eds.), Handbook of Labor Economics, 3, Elsevier Science.

Baker M., Fortin N.M. (2001), “Occupational gender composition and wages in Canada, 1987-1988", Canadian Journal of Economics, 34(2), pp. 345-376.

Bayard K., Hellerstein J., Neumark D. et K. Trotske (sous presse), "New evidence on gender segregation and gender differences in wages from matched employer-employee data" à paraître dans Journal of Labor Economics.

Becker G. (1971), The economics of discrimination, $2^{\text {nd }}$ edition, The University of Chicago Press.

Bergmann B.R. (1974), “Occupational segregation, wages and profits when employers discriminate by race or sex", Eastern Economic Journal, n 1, pp. 103-110.

Blinder A. (1973), "Wage discrimination - reduced form and structural estimates", Journal of Human Resources, 8(4), pp. 436-455.

Borghans L., Groot L. (1999), "Educational presorting and occupational segregation", Labour Economics $\mathrm{n}^{\circ} 6$, pp. 375-395.
Couppié T., Epiphane D. (2004), «Des bancs de l'école aux postes de travail... Chronique d'une ségrégation annoncée », Céreq, Notes Emploi Formation, $\mathrm{n}^{\circ} 6$.

Couppié T., Epiphane D. (2006), « la ségrégation des hommes et des femmes dans les métiers : entre héritage scolaire et construction sur le marché du travail », Formation Emploi $\mathrm{n}^{\circ} 93$, janvier-mars 2006.

Djellal F. (2002), « Le secteur du nettoyage face aux nouvelles technologies », Formation Emploi $\mathrm{n}^{\circ} 77$, janvier-mars, pp. 37-49.

Dupray A., Moullet S. (2004) «Femmes à l'entrée dans la vie active : un retard salarial en partie inexpliqué », Céreq, Notes Emploi Formation, n 12.

Dupray A. (2005), Eléments de cadrage sur les secondes parties de carrière: des observations aux concepts à mobiliser, séminaire d'ouverture du 22 novembre 2005 du projet Equallité-parcours d'avenir, Paris-Dauphine.

Epiphane D., Giret J.-F. Hallier P., Lopez A. et Sigot J.-C. (2001), « Génération 98 : à qui a profité l'embellie économique? », Céreq-Bref $\mathrm{n}^{\circ} 181$, décembre. 
Havet N., Lacroix G. (2002), Une modélisation des débuts de carrière et des écarts salariaux hommesfemmes, Communication aux JMA, Rennes, 6-7 juin 2002.

Karmel T., Maclachlan M., (1988) "Occupational sex segregation. Increasing or decreasing?" Economic Record 64 (186), pp. 187-195.

Killingsworth M. (1990), The economics of comparable worth, Kalamazoo, MI:upjohn institute of elmployment research.

Lemière S., Silvera R. (2001), "Approche de la compétence et genre : une analyse à partir des inégalités salariales et de l'évolution des emplois », Les Cahiers de l'IRETEP, $\mathrm{n}^{\circ} 3$, février.

Macpherson D., Hirsh B.T. (1995), "Wages and gender composition: why do women's job pay less?" Journal of Labor Economis, ${ }^{\circ}$ 13, pp. 426-471.

Meng X., Meurs D. (2001) «Différences de structure des emplois et écart salarial entre hommes et femmes en France », Économie et Prévision, ${ }^{\circ}{ }^{148}$, pp. 113-126.

Meurs D., Ponthieux S. (2000), «Une mesure de la discrimination dans l'écart de salaire entre hommes et femmes », Économie et statistique, $\mathrm{n}^{\circ} 337-388$, pp. 135-152.

Neumark D. (1988), "Employer's discriminatory behavior and the estimation of wage discrimination", Journal of Human Resources, ${ }^{\circ}$ 23, pp. 279-295.

Neumark D. (1999), "Wage differentials by race and sex: the roles of taste discrimination and labor market information", Industrial relations, vol. 38/3, pp. 414-445.
Oaxaca R.L. (1973), "Male-Female wage differentials in Urban Labor Markets", International Economic Review, vol. 14, pp. 693-709.

Oaxaca R.L., Ransom M.R. (1994), "On discrimination and the decomposition of wage differentials", Journal of Econometrics, vol. 61, pp. 5-21.

Okba M. (2004), «L'accès des femmes aux métiers : la longue marche vers l'égalité », Premières Synthèses, DARES, juillet.

Phelps E. (1972), « The stastical theory of racism ans sexism », American Economic Review, vol. 62, n 4 , pp. 649-661.

Pochic S. (2005), «Faire carrière: l'apport d'une approche en termes de genre », Formation Emploi $\mathrm{n}^{\circ}$ 91, juillet-septembre, pp. 75-93.

Rotschild, M. Stiglitz J. (1982), "A model of employment outcomes illustrating the effect of the structure of information on the level and distribution of income", Economic Letters, n ${ }^{\circ}$ 10, pp. 231-236.

Silber J. et Weber S. (1999), "Labour market discrimination: are there significant differences between the various decomposition procedures?", Applied Economics, vol. 31, pp. 359-365.

Sorensen E. (1990), "The crowding hypothesis and comparable worth issue: a survey and new results", Journal of Human Resources, $\mathrm{n}^{\circ}$ 25, p. 55-89.

Stiglitz J.E (1973), "Approaches to the Economics of Discrimination", American Economic Review, vol. $63, \mathrm{n}^{\circ} 2$, May.

Viprey M., Deroche L. (2000), « Conditions d'accès à l'entreprise des jeunes étrangers ou d'origine étrangère: nature des résistances », Migrations Etudes, $\mathrm{n}^{\circ} 94$, pp. 1-8. 


\title{
Résumé
}

\section{Les salaires des hommes et des femmes en début de vie active : des sources de disparité variables selon les professions}

\author{
Thomas Couppié, Arnaud Dupray, Stéphanie Moullet
}

Après trois ans de vie active, on analyse ici les disparités de salaire entre les hommes et les femmes selon la catégorie professionnelle, le degré de mixité des professions et l'origine de cette mixité : ségrégation professionnelle d'origine éducative ou/et liée au marché du travail. Ces disparités sont évaluées en termes de discrimination salariale. Les femmes, relativement aux hommes, sont les moins mal rémunérées dans les professions masculines, mais ce faible retard salarial cache une importante part injustifiée au regard des caractéristiques individuelles que l'on observe, et de ce fait assimilable à de la discrimination là caractéristiques égales, traitement différent). Dans les autres professions, la discrimination salariale accroît l'écart de salaire résultant des conditions d'emploi plus avantageuses pour les hommes. Par ailleurs, à degré de mixité donné, les professions dont la ségrégation est d'origine éducative sont porteuses de meilleurs niveaux de rémunération pour les femmes. Enfin, pour tous les groupes professionnels, la discrimination salariale à l'encontre des femmes relève plutôt de la situation professionnelle des individus que de leurs caractéristiques productives propres, comme l'éducation ou l'expérience professionnelle.

\section{Mots clés}

Division sexuelle du travail, inégalité salariale, profession, enquête Génération 98

Journal of Economic Literature : J16, J29, J31. 